\title{
Imaging targets moving in formation using parametric compensation
}

\author{
Jie Chen", Huaitie Xiao, Zhiyong Song and Hongqi Fan
}

\begin{abstract}
When conventional motion compensation algorithms that are fit for a single target are applied to cooperative targets imaging, a well-focused image cannot be obtained due to the low correlation between adjacent returned signals. In this paper, a parametric compensation method is proposed for the imaging of cooperative targets. First, the problem of the imaging is formulated by analyzing the translational motion of the target moving along a rectilinear fight path and by assuming a signal model of cooperative targets imaging. A bulk image is then obtained by parametric compensation of the linear and quadratic phase terms, which is performed by means of estimating the translational motion parameters through the fractional Fourier transform. Next, the number of targets in the bulk image is estimated by clustering number estimation, and the segmented images from the bulk image are separated by the normalized cuts. Finally, well-focused images are obtained by refined parametric compensation of the residual quadratic and cubic phase terms, which is carried out by estimating the parameters through maximizing the image contrast. Simulation results demonstrate the effectiveness of the proposed method.
\end{abstract}

Keywords: Formation fight; Radar imaging; Image segmentation; Parameter estimation; Keystone transform

\section{Introduction}

High-resolution radar imaging has been a widely addressed topic in recent years [1-3]. It has the ability to produce well-focused images under all weather conditions and day-and-night. When high-resolution radar imaging is applied to imaging of multiple targets moving in the same radar beam, the conventional motion compensation algorithms, which are suitable for a single moving target, cannot obtain a well-focused image due to the low correlation between adjacent returned signals [4-7]. Currently, high-resolution radar imaging of multiple targets has been an important issue in the radar imaging field.

The current techniques of multiple targets imaging can be categorized into two classes: direct imaging and separated imaging. The former method is based on timefrequency transformation $[2,8,9]$. However, this method has a heavy computational burden and needs to solve cross terms. The latter method is based on the separation of the returned signals. In this method, there are two basic techniques for signal separation. In the first type, the

\footnotetext{
* Correspondence: nudtatr_cj@163.com

ATR Key Laboratory, National University of Defense Technology, Changsha 410073, China
}

returned signal of each target is obtained by parameter separation [10-13]. However, this method is likely to fail when the differences between motion parameters are small in the formation flying. In the second type, the returned signals are separated from the region of target in the image domain $[14,15]$. However, the trajectory is coarsely estimated owing to without using phase information in [14] and the error of the first-order phase term compensation would be seriously increased when the blind velocity is large in [15].

For concise expression, multiple targets moving in a formation with almost identical velocity and acceleration are defined as cooperative targets. In the situation of cooperative target imaging, motion compensation for cooperative targets inherently confronts with difficulties due to the returned signals have low correlation as well as being overlapped in time. Therefore, motion compensation of the cooperative targets is important to the image quality. In order to reconstruct a well-focused image of cooperative targets, this paper proposes an imaging method using parametric compensation. The proposed method is carried out by three steps. In the first step, the translational motion of the target moving along a rectilinear fight path is analyzed and the translational

\section{穴}


motion parameters are estimated by means of the fractional Fourier transform (FrFT). The linear and quadratic phase compensations are then carried out to obtain a bulk image of the cooperative targets. The second step is image segmentation. In this step, the region of each target in the bulk image is determined by clustering number estimation and the normalized cuts. The third step consists in refined parametric compensation. In this step, well-focused images are obtained by refined compensation of the residual quadratic and cubic phase terms, which is carried out by estimating the parameters through maximizing the image contrast. Compared with the existing imaging methods by parameter separation, the proposed method can yield well-focused images of cooperative targets. Meanwhile, since the quadratic phase term is coarsely compensated and the cubic phase term is small, there is no large searching range for the residual quadratic and cubic parameters, which reduces the computational burden.

The remainder of this paper is organized as follows. Section 2 analyzes the translational motion and introduces the signal model of cooperative targets imaging. Section 3 describes the proposed imaging method. In Section 4, simulation results are presented to prove the effectiveness of the proposed method. Section 5 provides the conclusions.

\section{Signal model}

\subsection{Analysis of translational motion}

In the imaging interval, supposing the target is in uniformly accelerative rectilinear motion. At time $t=0$, the distance from the radar to the target is $R(0)$. By referring to the geometry shown in Figure 1, the distance between

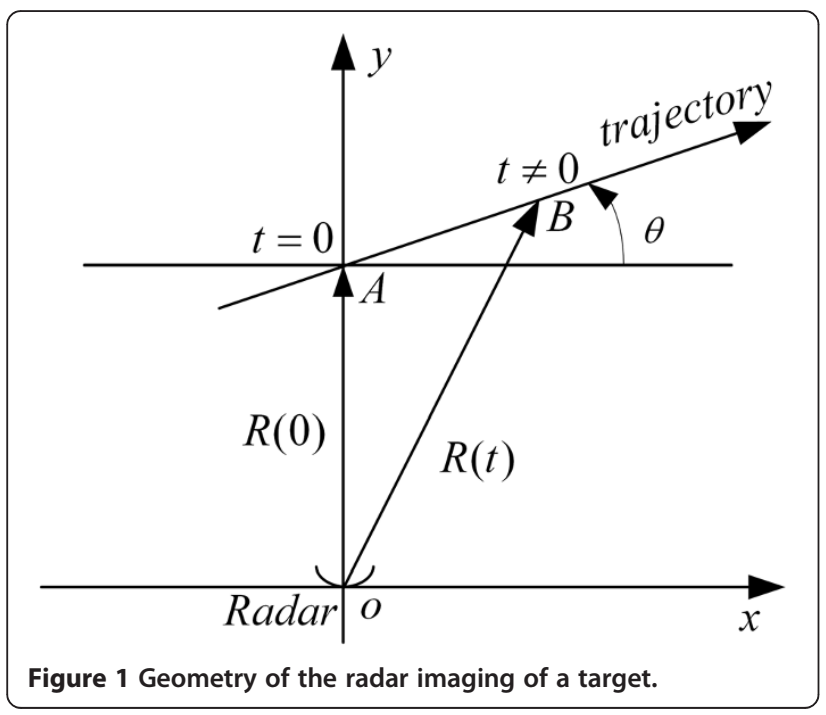

the target and the radar at a generic instant $t$ can be expressed as

$$
R(t)=\sqrt{\begin{array}{r}
\left(R(0)+v t \sin \theta+\frac{1}{2} a t^{2} \sin \theta\right)^{2} \\
+\left(v t \cos \theta+\frac{1}{2} a t^{2} \cos \theta\right)^{2},
\end{array}}
$$

where $v$ and $a$ denote the target's velocity and acceleration, respectively.

The target's radial velocity $v_{R}(t)$ and acceleration $a_{R}(t)$ can be expressed as

$$
\left\{\begin{array}{l}
v_{R}(t)=\dot{R}(t) \approx v \sin \theta+\left(a \sin \theta+\frac{v^{2} \cos ^{2} \theta}{R(0)}\right) t \\
a_{R}(t)=\ddot{R}(t) \approx a \sin \theta+\frac{v^{2} \cos ^{2} \theta}{R(0)}
\end{array}\right.
$$

When $a=0$, that is to say, the target is in uniform rectilinear motion, Equation (2) is given by

$$
\left\{\begin{array}{l}
v_{R}(t)=v \sin \theta+\frac{v^{2} \cos ^{2} \theta}{R(0)} t \\
a_{R}(t)=\frac{v^{2} \cos ^{2} \theta}{R(0)}
\end{array} .\right.
$$

From Equations (2) and (3), it is worth noting that the translational motion of the target can be viewed as a uniformly accelerative rectilinear motion regardless of the target is in uniform rectilinear motion or in uniformly accelerative rectilinear motion. In the imaging interval, $R(t)$ can be approximated with its second-order Taylor polynomial calculated around $t=0$ and it can be expressed as

$$
R(t) \approx R(0)+v_{R 0} t+\frac{1}{2} a_{R 0} t^{2}
$$

where $v_{R 0}=\dot{R}(0)$ and $a_{R 0}=\ddot{R}(0)$ are the initial radial target's velocity and acceleration, respectively.

\subsection{Signal model}

The imaging geometry is shown in Figure 2. Coordinate $(U, V)$ is the radar coordinate system. The target is described in coordinate $(x, y)$ with its origin located at the geometric center of the target, called the target coordinate. A scatterer $P$ locates on the target. $(X, Y)$ is the coordinate system translated from the radar and is used to describe rotations of the target, $\phi$ is the azimuth angle of the target with respect to the $(U, V)$ coordinate.

When $\phi=0$, the range from the radar to the scatterer $P$ is $[8]$

$$
\begin{aligned}
R_{P}(t) & =R(t)+x \cos (\theta(t))-y \sin (\theta(t)) \\
& \approx R(t)+x-y \omega t,
\end{aligned}
$$




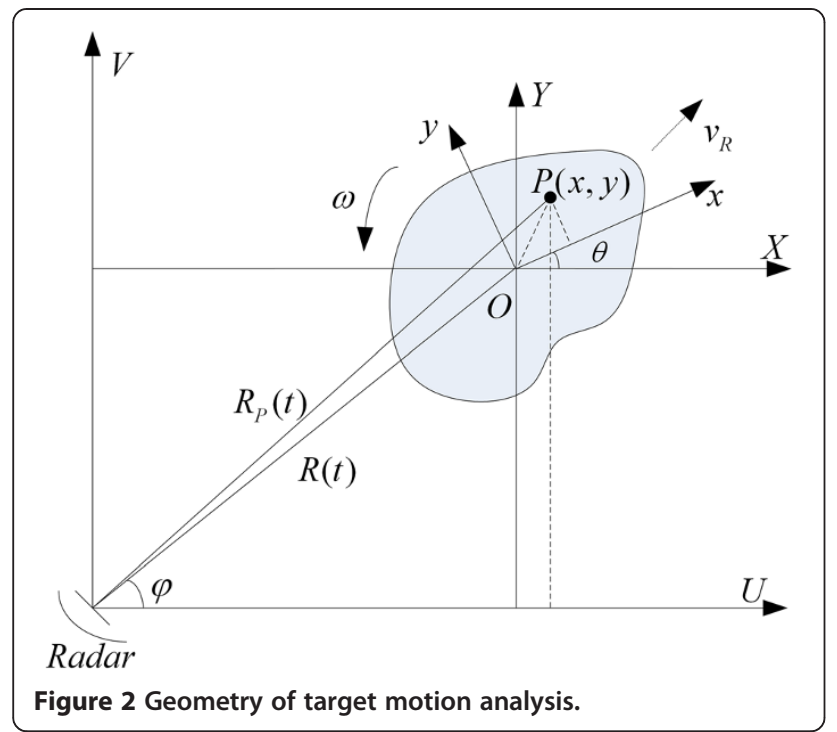

where $\omega$ is the target angular velocity in the imaging interval.

In the case of cooperative targets, we assume that the returned signals in fast-slow time can be expressed as

$$
\begin{aligned}
S_{R}\left(\hat{t}, t_{m}\right)= & \sum_{i=1}^{K} \sum_{j=1}^{q_{i}} A_{i, j} p\left(\frac{\hat{t}-2 R_{i, j}\left(t_{m}\right) / c}{T_{0}}\right) \operatorname{rect}\left(\frac{t_{m}}{T_{a}}\right) \\
& \exp \left(j 2 \pi\left(f_{c}\left(t-2 R_{i, j}\left(t_{m}\right) / c\right)\right),\right.
\end{aligned}
$$

where $p(t)=\operatorname{rect}\left(\frac{t}{T_{\ell}}\right) \exp \left(j \pi \gamma t^{2}\right)$ denotes the chirp pulse, $\gamma$ is the chirp rate, $t=t-t_{m}$ and $t_{m}=m T_{r}$ are known as the fast time and slow time, respectively, $K$ is the number of targets, $q_{i}$ is the number of scatterers of the target $i, A_{i, j}$ is the strength of the scatterer $j$ in the target $i, f_{c}$ is the carrier frequency, $R_{i, j}\left(t_{m}\right)=R_{i, 0}\left(t_{m}\right)+x_{i, j}-y_{i, j} \omega t_{m}$ is the distance between the radar and the scatterer $j$ of the target $i$, $T_{0}$ is the pulse length, $T_{a}$ is the imaging interval, $c$ is the light speed.

After downconversion, in the range frequency-slow time domain, Equation (6) can be expressed as

$$
\begin{aligned}
S_{R}\left(f, t_{m}\right)= & \sum_{i=1}^{K} \sum_{j=1}^{q_{i}} A_{i, j} \operatorname{rect}\left(\frac{f}{B}\right) \operatorname{rect}\left(\frac{t_{m}}{T_{a}}\right) \\
& \exp \left(-j \frac{4 \pi\left(f_{c}+f\right)}{c} R_{i, j}\left(t_{m}\right)\right),
\end{aligned}
$$

where $B$ is the bandwidth.
If Equation (4) is used in Equation (7), we obtain

$$
\begin{aligned}
S_{R}\left(f, t_{m}\right)= & \sum_{i=1}^{K} \sum_{j=1}^{q_{i}} A_{i, j} \operatorname{rect}\left(\frac{f}{B}\right) \operatorname{rect}\left(\frac{t_{m}}{T_{a}^{\prime}}\right) \\
& \exp \left(-j \frac{4 \pi\left(f_{c}+f\right)}{c}\left(R_{i, 0}(0)+v_{i, R 0} t_{m}\right.\right. \\
& \left.\left.+\frac{1}{2} a_{i, R 0} t_{m}^{2}+x_{i, j}-y_{i, j} \omega t_{m}\right)\right)
\end{aligned}
$$

For $f=f_{0}$, that is, in the special range cell, the Doppler frequency shift induced by the translational motion of the target $i$ is

$$
\begin{aligned}
f_{i, d}(t) & =-\frac{2\left(f_{c}+f_{0}\right)}{c}\left(v_{i, R 0}-y_{i, j} \omega+a_{i, R 0} t\right), \\
& =f_{i, d 0}+k_{i, 0} t
\end{aligned}
$$

where $f_{i, d 0}=-\frac{2\left(f_{c}+f_{0}\right)}{c}\left(v_{i, R 0}-y_{i, j} \omega\right)$ is the initial frequency, $k_{i, 0}=-\frac{2\left(f_{c}+f_{0}\right)}{c} a_{i, R 0}$ denotes the chirp rate. It is worth pointing out that the Doppler frequency shift induced by the translational motion is approximated as the linear frequency modulation signal (LFM signal) in range cell. If the initial frequency and the chirp rate are estimated, the translational motion parameters can also be coarsely estimated.

By performing the Fourier transformation on Equation (8) with respect to $t_{m}$ and applying the principle of stationary phase, we have

$$
\begin{gathered}
S_{R}\left(f, f_{\text {azi }}\right)=\sum_{i=1}^{K} \sum_{j=1}^{q_{i}} A_{i, j}^{\prime} \operatorname{rect}\left(\frac{f}{B}\right) \operatorname{rect}\left(\frac{f_{\text {azi }} c}{4 a_{i, R 0}\left(f_{c}+f\right) T_{a}}+\frac{v_{i, R 0}}{2 a_{i, R 0} T_{a}}\right) \\
\exp \left(-j\left(\frac{-\pi c}{4 a_{i, R 0}\left(f_{c}+f\right)} f_{\text {azi }}^{2}-\frac{\pi\left(v_{i, R 0}-y_{i, j} \omega\right)}{a_{i, R 0}} f_{\text {azi }}\right.\right. \\
\left.\left.\quad-\frac{\pi\left(v_{i, R 0}-y_{i, j} \omega\right)^{2}}{a_{i, R 0} c}\left(f_{c}+f\right)+\frac{4 \pi}{c}\left(R_{i, 0}(0)+x_{i, j}\right)\left(f_{c}+f\right)\right)\right) .
\end{gathered}
$$

The first phase term is the Doppler chirp term as well as the range-Doppler coupling term which should be compensated to eliminate the image defocusing. The second phase term is the Doppler term and reflects the cross range of the scatterer $j$ of the target $i$. The third phase term is the range shift term which should be compensated to reduce the image deformation. The fourth phase term is the range term and reflects the range of the scatterer $j$ of the target $i$. Therefore, in order to obtain a well-focused image, it is necessary to estimate the translational parameters and eliminate their influences on the image. 


\section{Imaging algorithm}

\subsection{Parameters estimation by the FrFT}

The FrFT of signal $x(t)$ with angle $\alpha$ is defined by [16]

$$
X_{\alpha}(u)=F_{\alpha}[x(t)]=\int_{-\infty}^{\infty} x(t) K_{\alpha}(t, u) d t
$$

where $K_{\alpha}(t, u)$ represents the transformation kernel which can be defined as

$$
K_{\alpha}(t, u)= \begin{cases}\sqrt{\frac{1-j \cot \alpha}{2 \pi}} \exp \left(j \frac{t^{2}+u^{2}}{2} \cot \alpha-j t u \csc \alpha\right) & \alpha \neq n \pi \\ \delta(t-u) & \alpha=2 n \pi \\ \delta(t+u) & \alpha=(2 n+1) \pi\end{cases}
$$

where $\delta(t)$ denotes the Dirac function.

Supposing that the LFM signal $x(t)$ is

$$
x(t)=A \exp \left(j 2 \pi\left(f_{d c} t+\frac{1}{2} k_{d} t^{2}\right)\right),
$$

where $f_{d c}$ and $k_{d}$ denote the initial frequency and the chirp rate, respectively. They can be estimated from the peak position of its fractional Fourier spectrum as follows

$$
\left\{\begin{array}{l}
{[\hat{\alpha}, \hat{u}]=\arg \max _{\alpha, u}\left|X_{\alpha}(u)\right|^{2}} \\
\hat{f}_{d c}=\hat{u} \csc \hat{\alpha} \\
\hat{k}_{d}=-\cot \hat{\alpha}
\end{array} .\right.
$$

In the case of cooperative targets imaging, it can be noted that the differences in radial velocities of different targets are small, and the radial accelerations of different targets can be considered as the same value from Equation (2). From Equation (9), it is known that the returned signals in range cell can be approximated as LFM signal. In the case of proper angle $\alpha$, the spectrum values of the returned signals in a range cell almost entirely reach a maximum owing to the almost identical radial acceleration. If we choose the strong spectrum value, the initial frequency and the chirp can be estimated by the FrFT. Meanwhile, the radial acceleration can be obtained as follows

$$
\hat{a}_{i, R 0}=-\frac{c}{2\left(f_{c}+f_{0}\right)} \hat{k}_{i, 0}
$$

Since $y_{i, j} \omega$ is always on the order of one [15], $\left(v_{i, R 0}-y_{i, j} \omega\right)$ can be approximated as $v_{i, R O}$. Consequently, the radial velocity can be coarsely estimated by

$$
\hat{v}_{i, R 0} \approx-\frac{c}{2\left(f_{c}+f_{0}\right)} \hat{f}_{i, d c}
$$

Therefore, the radial acceleration $\hat{a}_{R 0}$ and velocity $\hat{v}_{R 0}$ of the cooperative targets can be coarsely equated with $\hat{a}_{i, R 0}$ and $\hat{v}_{i, R 0}$, respectively.

\subsection{Parametric compensation}

Ignoring the higher order terms, the phase term in the imaging interval is a second-order polynomial in Equation (10). In order to obtain the bulk image of the cooperative targets, compensation of the linear and quadratic phase terms should be carried out to eliminate the influences of translational motion.

\subsubsection{The linear phase term compensation}

To eliminate the influence of the linear phase term, the radial velocity of the cooperative targets is used to the linear phase term compensation and the corresponding compensation term can be expressed as

$$
\phi_{1}=\exp \left(j \frac{4 \pi\left(f_{c}+f\right)}{c} \hat{v}_{R 0} t_{m}\right)
$$

After compensation, we obtain

$$
\begin{aligned}
S_{R}^{\prime}\left(f, t_{m}\right)= & \sum_{i=1}^{K} \sum_{j=1}^{q_{i}} A_{i, j} \operatorname{rect}\left(\frac{f}{B}\right) \operatorname{rect}\left(\frac{t_{m}}{T_{a}}\right) \\
& \exp \left(-j \frac{4 \pi\left(f_{c}+f\right)}{c}\left(R_{i, 0}(0)+\left(\Delta v_{i}-y_{i, j} \omega\right) t_{m}\right.\right. \\
& \left.+\frac{1}{2} a_{i, R 0} t_{m}^{2}+x_{i, j}\right),
\end{aligned}
$$

where $\Delta v_{i}=v_{i, R 0}-\hat{v}_{R 0}$ is the residual radial velocity, which still induces range shift. The keystone transform is then utilized to eliminate the influence of the residual radial velocity. The keystone transform is [17]

$$
t_{m}=\left(\frac{f_{c}}{f+f_{c}}\right) \tau_{m}
$$

If Equation (19) is used in Equation (18), we obtain

$$
\begin{gathered}
S_{R}^{\prime}\left(f, \tau_{m}\right)=\sum_{i=1}^{K} \sum_{j=1}^{q_{i}} A_{i, j} \operatorname{rect}\left(\frac{f}{B}\right) \operatorname{rect}\left(\frac{t_{m}}{T_{a}}\right) \\
\exp \left(-j \frac{4 \pi\left(f_{c}+f\right)}{c}\left(R_{i, j}(0)+x_{i, j}\right)\right) \\
\cdot \exp \left(-j \frac{4 \pi f_{c}}{c}\left(\Delta v_{i}-y_{i, j} \omega\right) \tau_{m}\right) \\
\exp \left(-j \frac{2 \pi}{c}\left(1+\frac{f}{f_{c}}\right)^{-1} f_{c} a_{i, R 0} \tau_{m}^{2}\right) .
\end{gathered}
$$

Thus, the linear coupling between $f$ and $t_{m}$ can be removed. However, residual coupling still exists in the quadratic phase term, which induces image defocusing and should be compensated. 


\subsubsection{The quadratic phase term compensation}

After the linear phase term compensation, the quadratic phase term can be compensated by the following expression

$$
\phi_{2}=\exp \left(j \frac{2 \pi f_{c} \hat{a}_{R 0} \tau_{m}^{2}}{c\left(1+f / f_{c}\right)}\right)
$$

Then, the returned signals can be written as follows

$$
\begin{gathered}
S_{R}^{\prime \prime}\left(f, \tau_{m}\right)=\sum_{i=1}^{K} \sum_{j=1}^{q_{i}} A_{i, j} \operatorname{rect}\left(\frac{f}{B}\right) \operatorname{rect}\left(\frac{t_{m}}{T_{a}}\right) \\
\exp \left(-j \frac{4 \pi\left(f_{c}+f\right)}{c}\left(R_{i, j}(0)+x_{i, j}\right)\right) \\
\cdot \exp \left(-j \frac{4 \pi f_{c}}{c}\left(\Delta v_{i}-y_{i, j} \omega\right) \tau_{m}\right) \\
\exp \left(-j \frac{2 \pi}{c}\left(1+\frac{f}{f_{c}}\right)^{-1} \Delta a_{i, R 0} \tau_{m}^{2}\right),
\end{gathered}
$$

where $\Delta a_{i, R 0}=a_{i, R 0}-\hat{a}_{R 0}$ denotes the residual radial acceleration. The last phase term in Equation (22) represents the uncorrected quadratic phase term which will induce image blur. However, this image blur can be negligible if the residual radial acceleration $\Delta a_{i, R 0}$ is such that $\Delta a_{i, R 0}$ $T_{a}^{2}<2 \lambda_{c}$, where $\lambda_{c}$ is the carrier wavelength [18]. In this situation, the image in range-Doppler domain is

$$
\begin{aligned}
I_{0}\left(r, f_{d}\right)= & \int_{-\infty}^{\infty} \int_{-\infty}^{\infty} S_{R}^{\prime \prime}\left(f, \tau_{m}\right) \exp \left(j \frac{4 \pi f}{c} r+j 2 \pi f_{d} \tau_{m}\right) d f d \tau_{m} \\
= & B T_{a} \sum_{i=1}^{K} \sum_{j=1}^{q_{i}} A_{i, j} \operatorname{sinc}\left(\frac{2 \pi B}{c}\left(r-R_{i, j}(0)-x_{i, j}\right)\right) \\
& \operatorname{sinc}\left(\pi T_{a}\left(f_{d^{-}}-\frac{2 \pi f_{c}}{c}\left(\Delta v_{i}-y_{i, j} \omega\right)\right)\right),
\end{aligned}
$$

where $r$ denotes range, $f_{d}$ denotes Doppler.

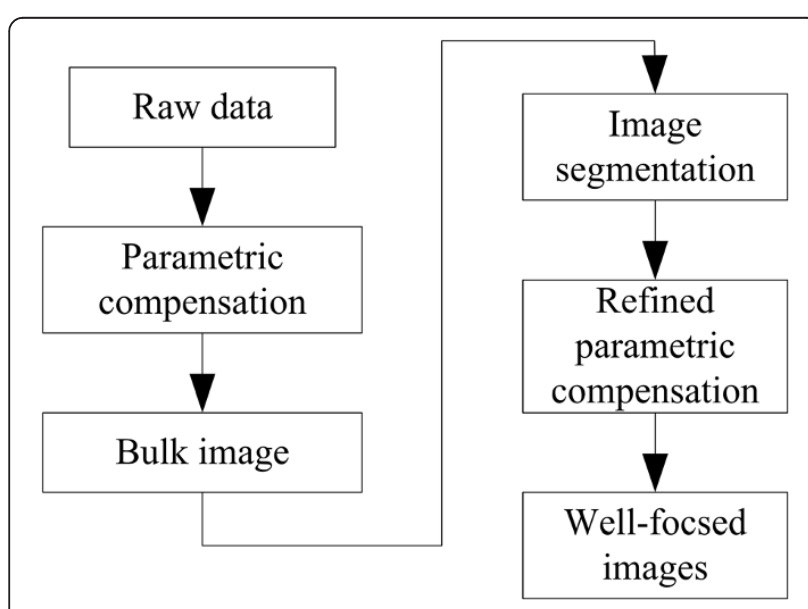

Figure 3 Flowchart of cooperative targets imaging.

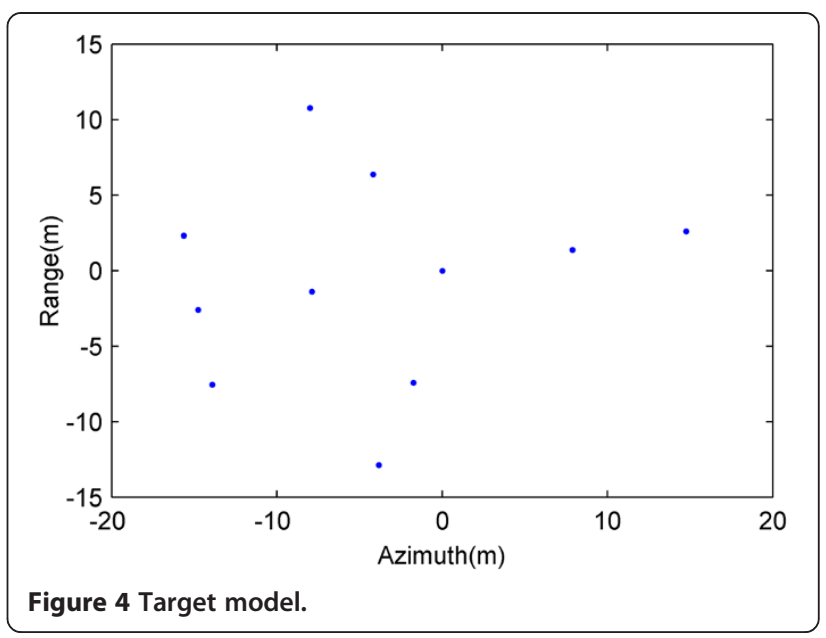

After compensation of the linear and quadratic phase terms, we can obtain the bulk image of cooperative targets and denote the absolute value of the image by $\left|I_{0}\left(r, f_{d}\right)\right|$.

\subsection{Image segmentation}

Since the residual quadratic and higher order phase terms are not entirely eliminated in the parametric compensation, the image is not well-focused and cannot be used to target identification. Refined parametric compensation is necessarily used to improve the image quality. Considering the differences in the residual quadratic and higher order motion parameters, refined parametric compensation should be carried out to target one by one. Therefore, in order to perform refined parametric compensation, the bulk image is segmented by clustering number estimation and the normalized cuts.

\subsubsection{Clustering number estimation}

Cooperative targets cannot generally be resolved in range domain. However, they can be resolved in azimuth

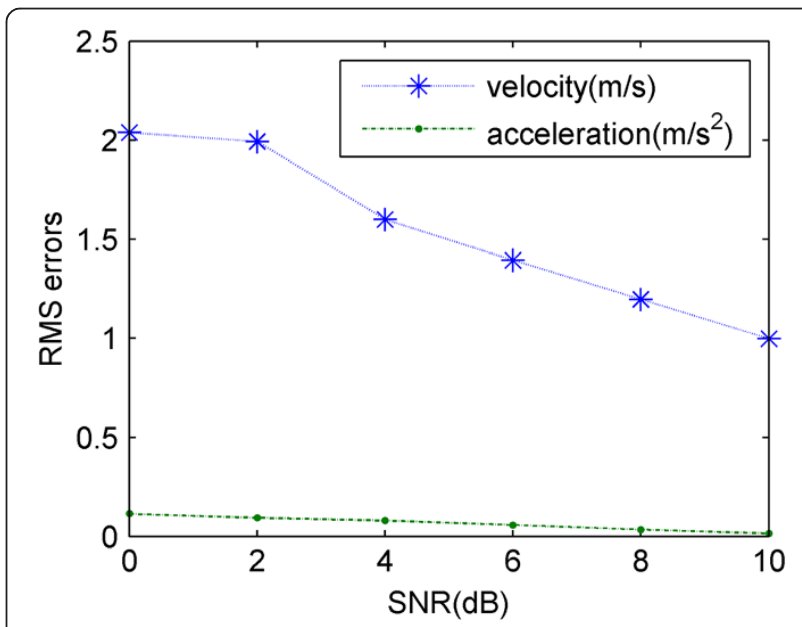

Figure 5 RMS errors of the estimated parameters of target 2. 


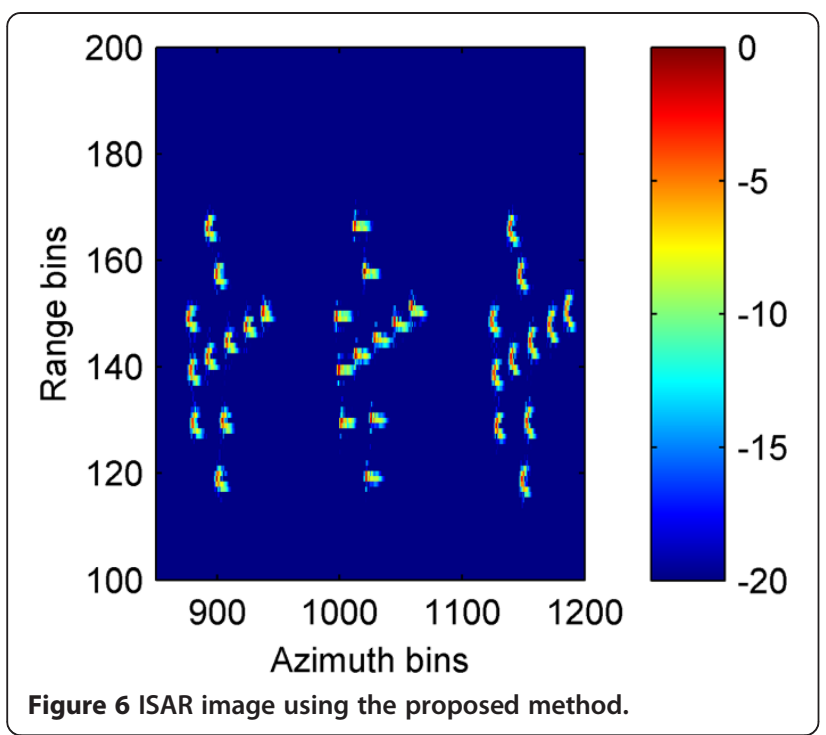

domain due to large distances exist in azimuth domain. According to estimating the number of the target centers in the bulk image, the clustering number can be determined as follows [15]

Step 1: Calculate normalized histograms of $\left|I_{0}\left(r, f_{d}\right)\right|$ along $r$ and $f_{d}$, respectively.

Step 2: For each histogram, obtain its smoothed envelope by low-pass filtering.

Step 3: Calculate the positions $\left\{\left(P_{r}, P_{f_{d}}\right)\right\}$ of local maxima of smoothed envelopes.

Step 4 . For each $\left(P_{r}, P_{f_{d}}\right)$, if the average of pixel values around it is above the threshold, it can be viewed as a true target center. Finally, the clustering number is equal to the number of true target centers.

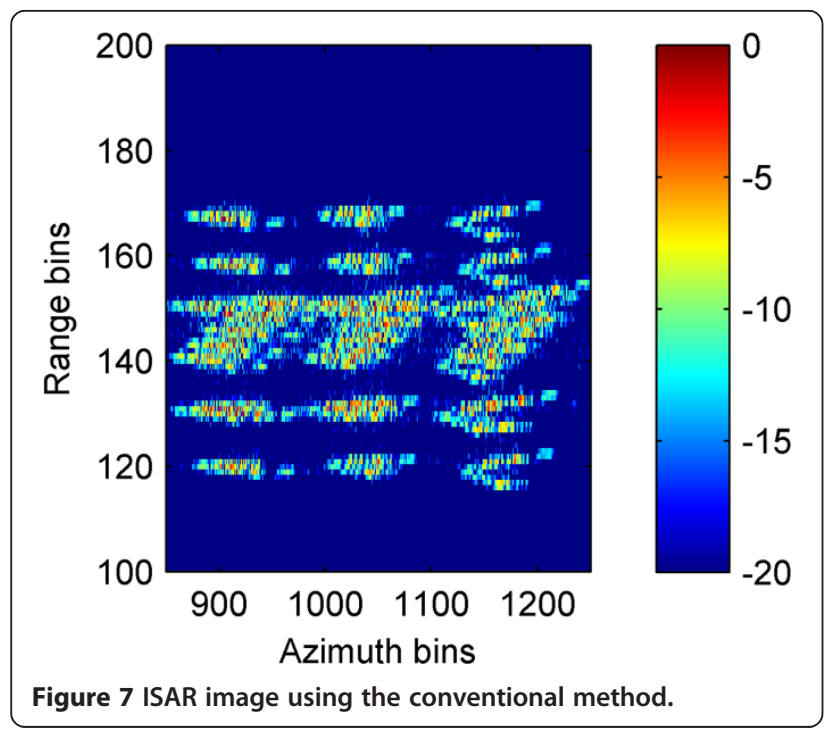

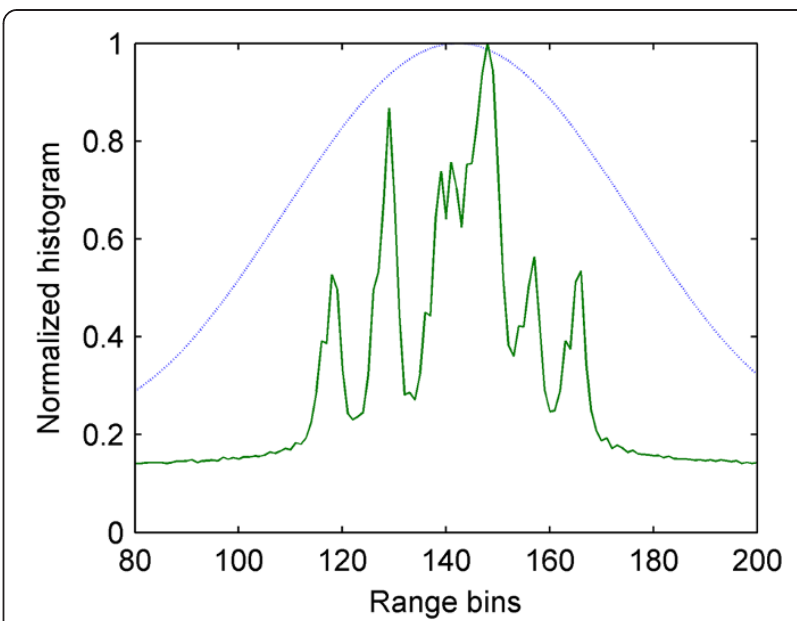

Figure 8 Normalized histogram and its envelope along range.

\subsubsection{Normalized cuts}

A graph $\mathbf{G}=(\mathbf{V}, \mathbf{E})$ is composed of two disjoint sets $A \cap$ $B=\varnothing, A \cup B=V$. In graph theoretic language, the degree of dissimilarity between these two sets is called the cuts

$$
\operatorname{cut}(A, B)=\sum_{u \in A, v \in B} w(u, v),
$$

where $w(u, v)$ is the graph edge weight between nodes $u$ and $v$. The normalized cuts $\operatorname{Ncut}(A, B)$ can be defined by [19]

$$
\begin{aligned}
\operatorname{Ncut}(A, B) & =\frac{\operatorname{cut}(A, B)}{\operatorname{assoc}(A, V)}+\frac{\operatorname{cut}(A, B)}{\operatorname{assoc}(B, V)} \\
= & \frac{\sum_{u \in A, v \in B} w(u, v)}{\sum_{u \in A, t \in V} w(u, t)}+\frac{\sum_{u \in A, v \in B} w(u, v)}{\sum_{u \in B, t \in V} w(u, t)} .
\end{aligned}
$$

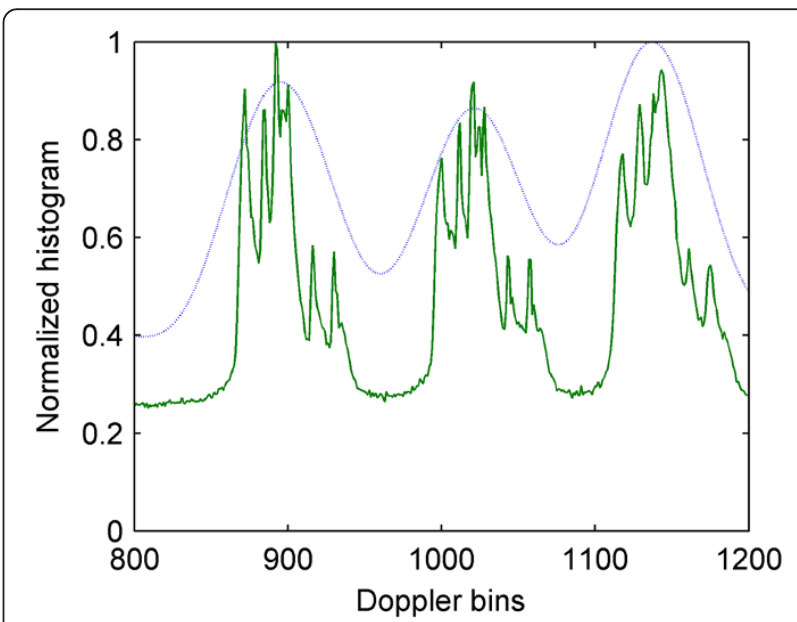

Figure 9 Normalized histogram and its envelope along azimuth. 
Table 1 Coordinates of potential target centers and the average of pixel values

\begin{tabular}{ccc}
\hline Center index & Coordinates & Average value \\
\hline 1 & $(143,895)$ & 0.6962 \\
2 & $(143,1,022)$ & 0.8173 \\
3 & $(143,1,137)$ & 0.8739 \\
\hline
\end{tabular}

Shi and Malik addressed that the optimal partition can be found by computing

$$
\begin{aligned}
\boldsymbol{y} & =\operatorname{argmin} \operatorname{Ncut}(A, B) \\
& =\underset{\boldsymbol{y}}{\operatorname{argmin}} \frac{\boldsymbol{y}^{T}(\boldsymbol{D}-\boldsymbol{W}) \boldsymbol{y}}{\boldsymbol{y}^{T} \boldsymbol{D} \boldsymbol{y}}
\end{aligned}
$$

where $y=\{a, b\}^{N}$ is a binary indicator vector and $y_{i}=a$ if pixel $i \in A$ and $y_{i}=b$ if pixel $j \in B$. N is the number of pixels. $\boldsymbol{W}$ is the association matrix with $W_{i j}=w(i, j) . \boldsymbol{D}$ is the diagonal matrix with $D_{i i}=\sum_{j} W_{i j}$.

According to the image segmentation, the region of each target can be determined from the bulk image and the range-slow time data of each target can be obtained by taking IFFT in each range bin of each segmented range-Doppler image. Therefore, refined parametric compensation for each target can be carried out to obtain a well-focused image.

\subsection{Enhancement of image}

The image contrast will reach a maximum if the image is well-focused [20]. Therefore, the image contrast can be utilized to compensate the residual second-order and higher order phase terms. Since the fourth-order phase term to the imaging quality can be ignorable [14], the compensation phase term can be written as follows

$$
\begin{aligned}
H^{\prime}\left(m T_{r}, \hat{\beta}, \hat{\gamma}\right)= & \exp \left(j \frac { 4 \pi } { c } f _ { c } ( 1 + \frac { f } { f _ { c } } ) ^ { - 1 } \left(\frac{1}{2} \hat{\beta}\left(m T_{r}\right)^{2}\right.\right. \\
& \left.\left.+\frac{1}{6} \hat{\gamma}\left(m T_{r}\right)^{3}\right)\right),
\end{aligned}
$$

where $\hat{\beta}$ is a estimation of residual radial acceleration $\beta$ and $\hat{\gamma}$ is a estimation of the radial jerk $\gamma$.

The image contrast is defined as follows

$$
C(\hat{\beta}, \hat{\gamma})=\frac{\sqrt{A\left\{\left[I_{0}^{2}\left(r, f_{d}, \hat{\beta}, \hat{\gamma}\right)-A\left\{I_{0}^{2}\left(r, f_{d}, \hat{\beta}, \hat{\gamma}\right)\right\}\right]^{2}\right\}}}{A\left\{I_{0}^{2}\left(r, f_{d}, \hat{\beta}, \hat{\gamma}\right)\right\}},
$$

where $I_{0}\left(r, f_{d}, \hat{\beta}, \hat{\gamma}\right)$ is obtained by compensating the returned signals through Equation (27), the operator $A(\cdot)$ denotes the image spatial mean. When $C(\hat{\beta}, \hat{\gamma})$ reaches a maximum, it indicates that $\beta$ and $\gamma$ are accurately estimated and then the image can be well-focused. According to the above imaging process, the flowchart of cooperative targets imaging is shown in Figure 3.

\subsection{Computational complexity analyses}

In this part, the computational complexity of major steps in the proposed method will be analyzed in terms of the number of operation. Suppose that the number of pulses, range sampling cells, searching residual radial acceleration, searching the radial jerk, and searching range frequency cells are $N, M, N_{\beta}, N_{\gamma}$ and $W$, respectively. As to the FrFT, WMN multiplications and $W M(N-1)$ additions are needed and the computational complexity is $O$ $(W M N)$. For the parametric compensation, $2 M N$ multiplications and $2 M(N-1)$ additions are needed and the computational complexity is $O(M N)$. As to the image segmentation, the computational complexity is $O(k M N)$, where $k$ is the number of steps Lanczos takes to converge. As to the image contrast, $N_{\beta} N_{\gamma}(8 M N+2 M N \log$ $\left.{ }_{2} M N\right)$ multiplications and $N_{\beta} N_{\gamma}\left(4 M N+3 M N \log _{2} M N\right)$ additions are needed and the computational complexity is $O\left(M N \log _{2} M N\right)$.

\section{Simulation results}

In this section, the returned signals of three targets separated along the $x$-axis on the same $x y$ plane are generated to prove the effectiveness of the proposed method.
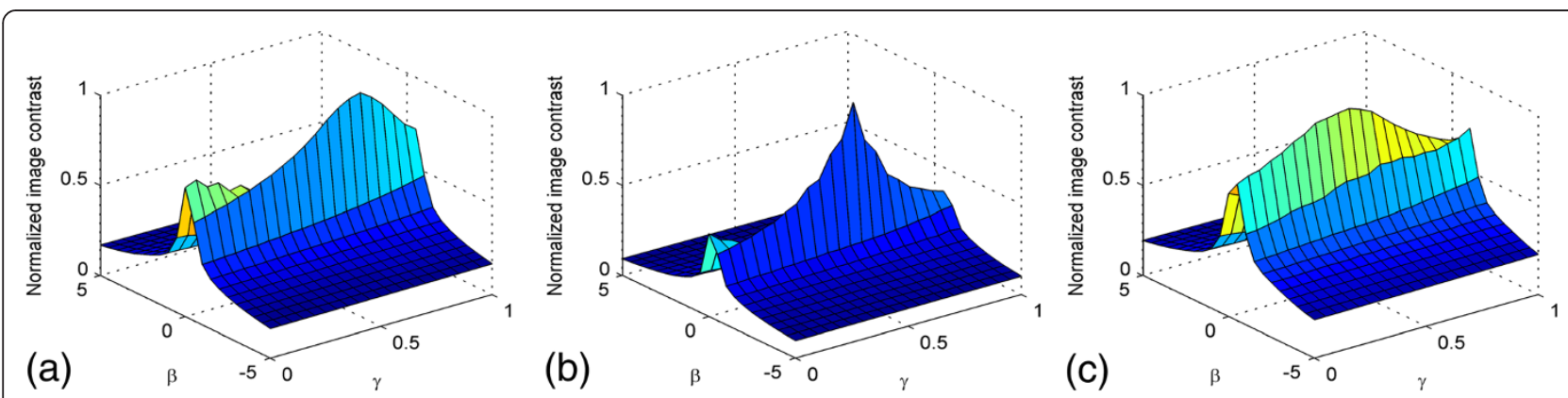

Figure 10 Image contrast. (a) Image contrast of target 1; (b) image contrast of target 2; (c) image contrast of target 3. 

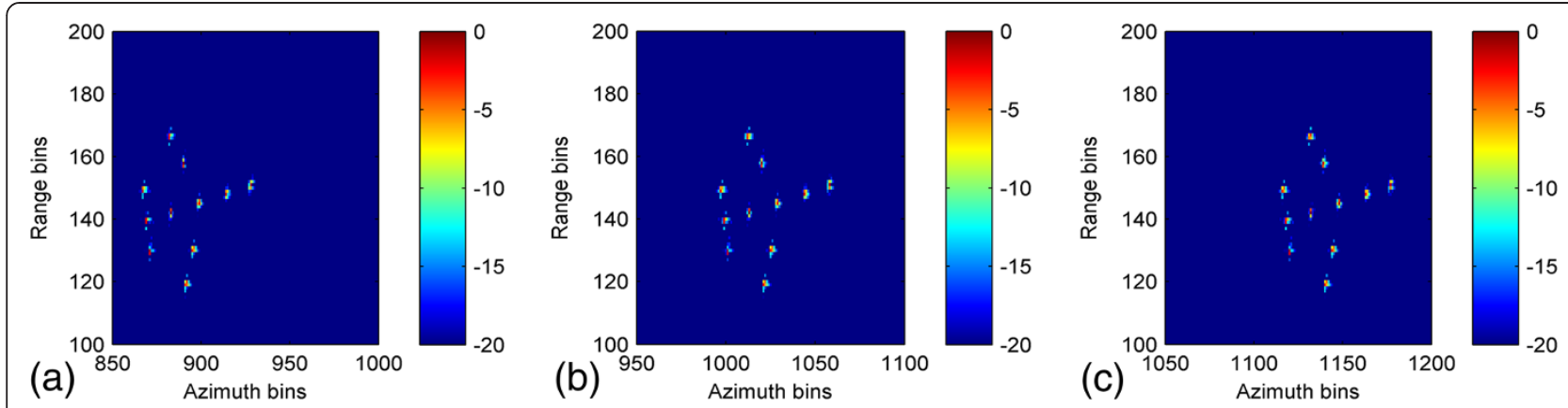

Figure 11 Well-focused images with SNR = $10 \mathrm{~dB}$. (a) ISAR image of target 1; (b) ISAR image of target 2; (c) ISAR image of target 3 .

We assume that the initial velocity and acceleration of three targets moving in a formation are $v_{1}=268 \mathrm{~m} / \mathrm{s}, a_{1}=$ $11 \mathrm{~m} / \mathrm{s}^{2}, v_{2}=270 \mathrm{~m} / \mathrm{s}, a_{2}=10 \mathrm{~m} / \mathrm{s}^{2}, v_{3}=272 \mathrm{~m} / \mathrm{s}$, and $a_{3}$ $=9 \mathrm{~m} / \mathrm{s}^{2}$, respectively. The angle $\theta$ is $10^{\circ}$. Target 1 , target 2 , and target 3 are located at $(-65 \mathrm{~m}, 0 \mathrm{~m}),(-10 \mathrm{~m}, 0 \mathrm{~m})$, and $(40 \mathrm{~m}, 0 \mathrm{~m})$, respectively. The radar is located at $(0 \mathrm{~m}$, $-8,000 \mathrm{~m}$ ). The pulse repetition frequency (PRF) is 2,000 $\mathrm{Hz}$, the carried frequency is $9 \mathrm{GHz}$, the bandwidth is 300 $\mathrm{MHz}$, the number of sampling data is 1,024 and the echo number for coherent imaging processing is 2,000 . The target model is shown in Figure 4. Complex white Gaussian noise is added to the returned signals.

Monte Carlo simulation was performed to verify the performance of the parameter estimated method. The number of Monte Carlo simulation is 500. The rootmean-square errors (RMS errors) of the radial velocity and acceleration of the target 2 are shown in Figure 5. It can be seen from Figure 5 that the RMS errors of the estimated radial velocity is large, while that of the estimated radial acceleration are close to zero with different SNRs. The reason is that the radial acceleration can be accurately estimated and the radial velocity was coarsely estimated. Fortunately, the influence of the residual of the radial velocity can be eliminated by the keystone transform. When the signal-to-noise ratio (SNR) is 10 $\mathrm{dB}$, the averages of the radial velocity and acceleration estimated by the FrFT are $45.6752 \mathrm{~m} / \mathrm{s}, 10.5218 \mathrm{~m} / \mathrm{s}^{2}$, respectively.
After the linear and quadratic phase term compensations, a resolved bulk image is shown in Figure 6. When applying the conventional imaging method using envelope cross-correlation and the phase gradient autofocus algorithm, this method yields an image in Figure 7. It is evident that the image in Figure 7 does not yield a resolved image, which demonstrates the conventional imaging method does not work well for cooperative targets imaging.

Figures 8 and 9 show normalized histograms along range and azimuth by solid line and their envelops by dashed line, respectively. According to Figures 8 and 9, coordinates of local maxima of envelops are $(143,895)$, $(143,1,022)$, and $(143,1,137)$, which are likely to the target centers. Table 1 lists coordinates of potential target centers and the average of pixel values around each coordinate of local maxima. According to the normalized average, the number of target centers is three, so the clustering number is three.

After image segmentation, image contrasts of the segmented image with $\beta$ and $\gamma$ are shown in Figure 10a,b,c, respectively, where $\beta \in[-5,5], \gamma \in[0,1]$ [14]. It is important to note that the image contrast is mainly affected by $\beta$, which indicates that the quadratic coefficient plays an important role in the imaging quality. After refined parametric compensation by $(\beta, \gamma)$ corresponding to the image contrast maximization, the enhanced images of three targets in decibel are shown in Figure 11a,b,c, respectively. It
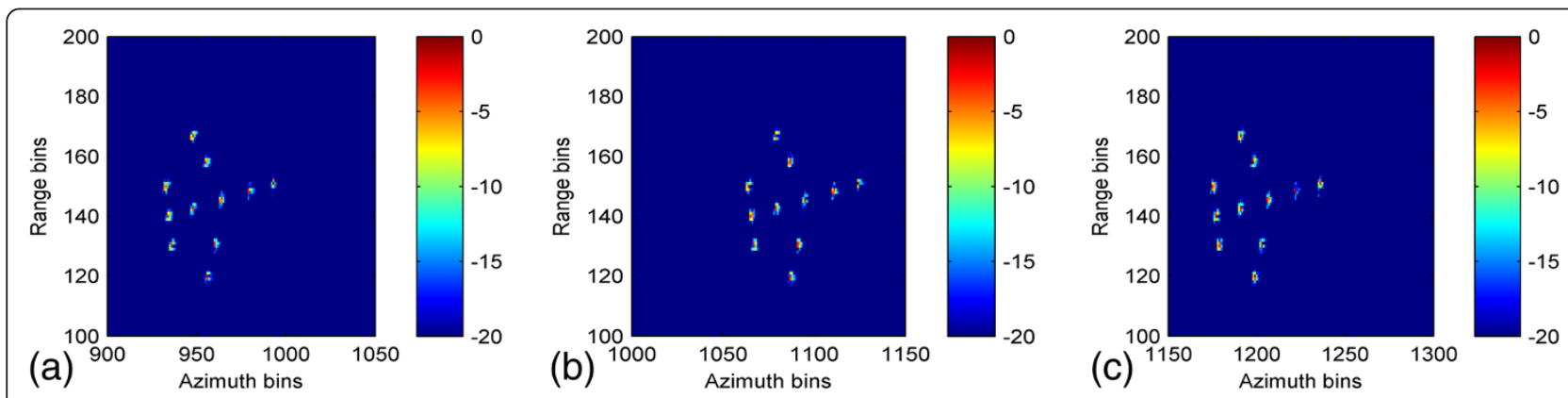

Figure 12 Well-focused images with SNR $=0 \mathbf{d B}$. (a) ISAR image of target 1; (b) ISAR image of target 2; (c) ISAR image of target 3 . 
is evident that each image has better focus than its original image in Figure 6.

When the SNR is $0 \mathrm{~dB}$, the averages of the radial velocity and acceleration estimated by the FrFT are 49.8649 $\mathrm{m} / \mathrm{s}, 10.3582 \mathrm{~m} / \mathrm{s}^{2}$, respectively. The well-focused images of three targets in decibel are shown in Figure 12a,b,c, respectively. From this simulation, we believe that the proposed method can still image cooperative targets in the low SNR scenario.

\section{Conclusions}

In this paper, we have proposed a method to solve the radar imaging problem of cooperative targets. The method utilizes the parametric compensation of the linear and quadratic phase terms to obtain a bulk image. Image segmentation is used to separate the bulk image into different images. Refined parametric compensation of the residual quadratic and cubic phase terms is then carried out to obtain well-focused images. The simulation results demonstrate that the proposed method can successfully image cooperative targets.

In future work, an experiment to generate well-focused images of cooperative targets will be conducted based on measured data. Furthermore, our further study will focus on the imaging of cooperative targets that have more complicated motion forms.

\section{Competing interests}

The authors declare that they have no competing interests.

\section{Acknowledgements}

The authors are grateful to the anonymous reviewers for the provided feedback. This work was supported by the National Natural Science Foundation of China under grant no. 61372159.

Received: 2 September 2013 Accepted: 18 January 2014 Published: 24 January 2014

\section{References}

1. Q Zhang, YQ Jin, Aspects of radar imaging using frequency-stepped chirp signals. EURASIP J. Adv. Sig. Pr. 2006, 43-43 (2006)

2. VC Chen, Q Shie, Joint time-frequency transform for radar range-Doppler imaging. IEEE T Aero. Elec. Sys. 34, 486-499 (1998)

3. L Zhang, JL Sheng, J Duan, MD Xing, Z-J Qiao, Z Bao, Translational motion compensation for ISAR imaging under low SNR by minimum entropy. EURASIP J. Adv. Sig. Pr. 2013, 1-19 (2013)

4. CC Chen, CC Andrews, Target-motion-induced radar imaging. IEEE T Aero. Elec. Sys. 16, 1-14 (1980)

5. T Itoh, H Sueda, Y Watanabe, Motion compensation for ISAR via centroid tracking. IEEE T Aero. Elec. Sys. 32, 1191-1197 (1996)

6. J Li, R Wu, VC Chen, Robust autofocus algorithm for ISAR imaging of moving targets. IEEE T aero. Elec. Sys. 37, 1056-1069 (2001)

7. H Wu, D Grenier, GY Delisle, DG Fang, Translational motion compensation in ISAR image processing. IEEE Trans. Image Pr. 4, 1561-1571 (1995)

8. VC Chen, Time-frequency transforms for radar imaging and signal analysis (Artech House Radar Library, Boston, 2002)

9. VC Chen, ZZ Lu, Radar imaging of multiple moving targets, in Proceedings of the SPIE Radar Processing, Technology, and Application (San Diego, 1997). 31 July-1 August

10. YN Li, YW Fu, X Li, L Le, L Wei, ISAR imaging of multiple targets using particle swarm optimisation-adaptive joint time frequency approach. IET Sig. Pr. 4, 343-351 (2010)
11. K Yamamoto, M Iwamoto, T Fujisaka, An ISAR imaging algorithm for multiple targets of different radial velocity. ELECTR Commun. JPN 86, 1-10 (2003)

12. SH Park, KK Park, JH Jung, HT Kim, KT Kim, ISAR imaging of multiple targets using edge detection and Hough transform. J. Electromagnet. Wave. 2, 365-373 (2008)

13. G Choi, S Park, H Kim, K Kim, ISAR imaging of multiple targets based on particle swarm optimization and Hough transform. J. Electromagnet. Wave. 23, 1825-1834 (2009)

14. SH Park, HT Kim, KT Kim, Segmentation of ISAR images of targets moving in formation. IEEE T Geosci. Remote. 48, 2099-2108 (2010)

15. XR Bai, F Zhou, MD Xing, Z Bao, A novel method for imaging of group targets moving in a formation. IEEE T Geosci. Remote. 50, 221-231 (2012)

16. E Sejdić, I Djurović, L Stanković, Fractional Fourier transform as a signal processing tool: an overview of recent developments. Sig Pr. 91, 1351-1369 (2011)

17. RP Perry, RC DiPietro, RL Fante, SAR imaging of moving targets. IEEE T Aero. Elec. Sys. 35, 188-200 (1999)

18. G Franceschetti, J Tatoian, B Dutt, Aberrations in the SAR image of a moving target. Alta. Frequenza. 58, 175-183 (1989)

19. J Shi, J Malik, Normalized cuts and image segmentation. IEEE T Pattern Anal. 22, 888-905 (2000)

20. M Martorella, F Berizzi, B Haywood, Contrast maximisation based technique for 2-D ISAR autofocusing. IET Sig. Pr. 152, 253-262 (2005)

doi:10.1186/1687-6180-2014-8

Cite this article as: Chen et al:: Imaging targets moving in formation using parametric compensation. EURASIP Journal on Advances in Signal Processing 2014 2014:8.

\section{Submit your manuscript to a SpringerOpen ${ }^{\odot}$ journal and benefit from:}

- Convenient online submission

- Rigorous peer review

- Immediate publication on acceptance

- Open access: articles freely available online

- High visibility within the field

- Retaining the copyright to your article

Submit your next manuscript at $>$ springeropen.com 